\title{
Polynomial graph invariants and the KP hierarchy
}

\author{
Sergei Chmutov, Maxim Kazarian†, Sergey Lando
}

\begin{abstract}
We prove that the generating function for the symmetric chromatic polynomial of all connected graphs satisfies (after appropriate scaling change of variables) the Kadomtsev-Petviashvili integrable hierarchy of mathematical physics. Moreover, we describe a large family of polynomial graph invariants giving the same solution of the KP. In particular, we introduce the Abel polynomial for graphs and show this for its generating function. The key point here is a Hopf algebra structure on the space spanned by graphs and the behavior of the invariants on its primitive space.
\end{abstract}

\section{Introduction}

The Kadomtsev-Petviashvili (KP) hierarchy is an infinite system of nonlinear partial differential equations for a function $F\left(p_{1}, p_{2}, \ldots\right)$ of infinitely many variables, see Sec. 4 for details. The equations are indexed by partitions of integers $n, n \geq 4$, into two parts none of which is 1 . The first two equations, those corresponding to partitions of 4 and 5, are

$$
\begin{gathered}
\frac{\partial^{2} F}{\partial p_{2}^{2}}=\frac{\partial^{2} F}{\partial p_{1} \partial p_{3}}-\frac{1}{2}\left(\frac{\partial^{2} F}{\partial p_{1}^{2}}\right)^{2}-\frac{1}{12} \frac{\partial^{4} F}{\partial p_{1}^{4}} \\
\frac{\partial^{2} F}{\partial p_{2} \partial p_{3}}=\frac{\partial^{2} F}{\partial p_{1} \partial p_{4}}-\frac{\partial^{2} F}{\partial p_{1}^{2}} \cdot \frac{\partial^{2} F}{\partial p_{1} \partial p_{2}}-\frac{1}{6} \frac{\partial^{4} F}{\partial p_{1}^{3} \partial p_{2}}
\end{gathered}
$$

*Ohio State University, chmutov@math.osu.edu

${ }^{\dagger}$ Steklov Mathematical Institute RAS, National Research University Higher School of Economics, Skolkovo Institute of Science and Technology, kazarian@mccme.ru

${ }^{\ddagger}$ National Research University Higher School of Economics, Skolkovo Institute of Science and Technology, lando@hse.ru 
The left hand side of the equations correspond to partitions into two parts none of which is 1 , while the terms on the right hand sides correspond to partitions of the same number $n$ involving 1 . For $n=6$, there are two equations, which correspond to the partitions $2+4=6$ and $3+3=6$, and so on.

It is well known now [O00] that the generating function for simple connected Hurwitz numbers is a solution to the Kadomtsev-Petviashvili hierarchy. Simple connected Hurwitz numbers enumerate certain connected embedded graphs, and similar statements are known to be true for generating functions enumerating other kinds of embedded graphs as well. In the present paper, we derive a similar statement for the generating functions for Stanley's symmetric chromatic polynomial of graphs, the Abel polynomials enumerating rooted forests in graphs, and for a more general family of polynomial graph invariants. As far as we know, this is the first appearance of KP solutions coming from graph polynomials.

The symmetric chromatic polynomial. In 1995, Richard Stanley [St95] introduced the symmetric chromatic polynomial $X_{G}\left(x_{1}, x_{2}, \ldots\right)$ of a finite graph $G$ with the vertex set $V(G)$ as the sum over all proper colorings $\kappa: V(G) \rightarrow \mathbb{N}$ that color the endpoints of each edge into distinct colors:

$$
X_{G}\left(x_{1}, x_{2}, \ldots\right)=\sum_{\kappa} \prod_{v \in V(G)} x_{\kappa(v)} .
$$

Being a symmetric function in the variables $x_{1}, x_{2}, \ldots$, the function $X_{G}$ can be expressed through a basis of symmetric functions.

In fact, a polynomial $W_{G}\left(q_{1}, q_{2}, \ldots\right)$, equivalent to the expression of $X_{G}\left(p_{1}, p_{2}, \ldots\right)$ in terms of the power-sum functions $p_{m}:=\sum_{i=1}^{\infty} x_{i}^{m}$, was introduced in 1994 in [CDL94], motivated by some problems in knot theory. The equivalence was noted in [NW99, Theorem 6.1]:

$$
(-1)^{|V(G)|} W_{G}\left(q_{j}=-p_{j}\right)=X_{G}\left(p_{1}, p_{2}, \ldots\right) .
$$

Following [CDL94], we call $W_{G}\left(q_{1}, q_{2}, \ldots\right)$ the weighted chromatic polynomial. There are two advantages of using the [CDL94] approach as opposed to the [St95] one. Firstly, $W_{G}\left(q_{1}, q_{2}, \ldots\right)$ satisfies a contraction-deletion relation, which is understood properly in terms of graphs with weighted vertices. Secondly, $W_{G}\left(q_{1}, q_{2}, \ldots\right)$ encodes the graph $G$ as an element of an appropriate Hopf algebra in terms of its primitive elements. We give the precise definitions and review the relevant results in Sec.3.

The main result of this paper is that after an appropriate rescaling of the 
variables, the function

$$
\mathcal{W}\left(q_{1}, q_{2}, \ldots\right):=\sum_{G \text { connected }} \frac{W_{G}\left(q_{1}, q_{2}, \ldots\right)}{|\operatorname{Aut}(G)|}
$$

becomes a solution to the KP hierarchy. Here and below $|\operatorname{Aut}(G)|$ denotes the order of the automorphism group of the graph $G$. Note that we do not include the empty graph in the set of connected graphs.

The Abel polynomial. The classical sequence of Abel polynomials is defined as $x(x-a n)^{n-1}$. We consider its specialization with the parameter $a=-1, A_{n}(x):=x(x+n)^{n-1}$. This is a sequence of polynomials of degree $n=\operatorname{deg} A_{n}, n=0,1,2, \ldots$, with leading coefficient 1 and zero free term (for $n>0)$. It possesses the following remarkable properties:

- $\frac{d}{d x} e^{-d / d x}\left(A_{n}(x)\right)=n A_{n-1}(x)$, see [RST97];

- it is of binomial type [Ab826], $A_{n}(x+y)=\sum_{k=0}^{n}\left(\begin{array}{l}n \\ k\end{array}\right) A_{k}(x) A_{n-k}(y)$;

- its coefficient of $x^{k}$ is equal to the number of rooted spanning forests consisting of $k$ trees (each of which has a root) in the complete graph $K_{n}$. For instance, the coefficient of $x$ equals $n^{n-1}$, the number of rooted spanning trees of $K_{n}$, according to the famous Cayley formula.

The last property allows us to define the Abel polynomial of an arbitrary graph and make it into a polynomial of several variables $q_{1}, q_{2}, \ldots$ Namely, with each spanning forest of a graph $G$, we associate a monomial equal to the product of $\left(i \cdot q_{i}\right)$ over the trees of the forest, where $i$ stands for the number of vertices of the tree, and the multiplication by $i$ corresponds to the different choices of the root of the tree. The Abel polynomial $A_{G}\left(q_{1}, q_{2}, \ldots\right)$ of $G$ is equal to the sum of these monomials over all (non-rooted) spanning forests of $G$.

In particular, for a graph $G$ with $n$ vertices, the coefficient of $q_{n}$ in $A_{G}$ is equal to the number of rooted spanning trees of $G$. For a complete graph $K_{n}$, after the substitution $q_{i}=x$ for all $i$, the polynomial becomes the Abel polynomial $A_{n}(x), A_{K_{n}}(x, x, \ldots)=A_{n}(x)$. This polynomial is related to the spectrum of the combinatorial Laplace operator via the matrix-forest theorem [CS06, Kn13].

An analog of the second property of the Abel polynomial relates it to the Hopf algebra structure on graphs. We discuss it in detail in Sec.3. As a consequence, we get one more result: after an appropriate rescaling of the variables, the function

$$
\mathcal{A}\left(q_{1}, q_{2}, \ldots\right):=\sum_{G \text { connected }} \frac{A_{G}\left(q_{1}, q_{2}, \ldots\right)}{|\operatorname{Aut}(G)|}
$$


becomes a solution to the KP hierarchy.

The work on this paper started during the second author's visit to the Ohio State University, to which he expresses his gratitude. The second and the third authors appreciate the support of RSF grant, project 16-11-10316 dated 11.05.2016.

\section{Main results}

The weighted chromatic polynomial.

Necessary definitions will be given in Sec. 3. Besides the function $\mathcal{W}\left(q_{1}, q_{2}, \ldots\right)$, we introduce the function

$$
\mathcal{W}^{\circ}\left(q_{1}, q_{2}, \ldots\right):=\exp (\mathcal{W})=\sum_{G} \frac{W_{G}\left(q_{1}, q_{2}, \ldots\right)}{|\operatorname{Aut}(G)|}
$$

represented by the sum over all (not necessarily connected, possibly empty) simple graphs.

For rescaling the variables we need the sequence [A134531]

$$
c_{1}=1 ; \quad c_{n}=(-1)^{n+1}\left[1+\sum_{k=1}^{n-1}(-1)^{k} 2^{k(n-k)}\left(\begin{array}{c}
n-1 \\
k-1
\end{array}\right) c_{k}\right] .
$$

In particular, $c_{1}=1, \quad c_{2}=1, \quad c_{3}=5, \quad c_{4}=79, \quad c_{5}=3377, \ldots$

Theorem 2.1 After the substitution $q_{n}=\frac{2^{n(n-1) / 2}(n-1) !}{c_{n}} \cdot p_{n}$, the generating function $\mathcal{W}$ becomes a solution to the KP hierarchy of partial differential equations and $\mathcal{W}^{\circ}$ becomes a $\tau$-function of the KP hierarchy.

The Abel polynomial.

Similarly, we introduce the function

$$
\mathcal{A}^{\circ}\left(q_{1}, q_{2}, \ldots\right):=\exp (\mathcal{A})=\sum_{G} \frac{A_{G}\left(q_{1}, q_{2}, \ldots\right)}{|\operatorname{Aut}(G)|},
$$

and the constants $a_{n}:=2^{(n-1)(n-2) / 2} n^{n-1}$ for $n=1,2, \ldots$, so that $a_{1}=1$, $a_{2}=2, a_{3}=18, a_{4}=512$.

Theorem 2.2 After the substitution $q_{n}=\frac{2^{n(n-1) / 2}(n-1) !}{a_{n}} \cdot p_{n}$, the generating function $\mathcal{A}$ becomes the solution to the KP hierarchy from Theorem 2.1. $S o \mathcal{A}^{\circ}$ becomes the $\tau$-function of the KP hierarchy from the same theorem. 
Theorems 2.1 and 2.2 are corollaries of the following more general statement concerning umbral graph polynomial invariants as defined in Sec. 3. Both the weighted chromatic polynomial and the Abel polynomial are examples of umbral graph invariants.

Theorem 2.3 Let I be an umbral graph polynomial invariant with values in the polynomial ring in infinitely many variables $q_{1}, q_{2}, \ldots$ Define two generating functions by

$$
\begin{aligned}
\mathcal{I}^{\circ}\left(q_{1}, q_{2}, \ldots\right) & =\sum_{G} \frac{I_{G}\left(q_{1}, q_{2}, \ldots\right)}{|\operatorname{Aut}(G)|} \\
\mathcal{I}\left(q_{1}, q_{2}, \ldots\right) & =\sum_{G \text { connected }} \frac{I_{G}\left(q_{1}, q_{2}, \ldots\right)}{|\operatorname{Aut}(G)|}
\end{aligned}
$$

where the first summation is carried over all graphs, while the second one over all connected graphs and $|\operatorname{Aut}(G)|$ denotes the order of the automorphism group of the graph $G$.

Then after an appropriate rescaling of the variables $q_{n}=$ $\frac{2^{n(n-1) / 2}(n-1) !}{i_{n}} \cdot p_{n}$, where $i_{n}, n=1,2, \ldots$, are some constants, the generating function $\mathcal{I}$ becomes a solution to the KP hierarchy of partial differential equations. After the same rescaling of the variables the generating function $\mathcal{I}^{\circ}$ becomes a $\tau$-function of the KP hierarchy.

The rescaling constants $i_{n}$ are given by the sum

$$
i_{n}=n ! \sum_{G \text { connected }} \frac{\left[q_{n}\right] I_{G}\left(q_{1}, q_{2}, \ldots\right)}{|\operatorname{Aut}(G)|}
$$

of the coefficients of $q_{n}$.

We would like to stress that, after the rescaling in the theorem, any umbral graph invariant $I$ leads to one and the same generating function.

In the last formula the summation can well be carried over all graphs rather than only connected ones, since the coefficient of $q_{n}$ in the value $I_{G}\left(q_{1}, q_{2}, \ldots\right)$ on any disconnected graph $G$ with $n$ vertices is 0 . Note also that the value of an umbral graph invariant $I_{G}$ on any graph with $n$ vertices is a weighted homogeneous polynomial of weight $n$, with weights of the variable $q_{i}$ taken to be $i$, for $i=1,2, \ldots$.

The previous theorem can be made more precise.

Theorem 2.4 After the rescaling of the variables described in Theorem 2.3, the generating function $\mathcal{I}^{\circ}$ becomes the following linear combination of onepart Schur polynomials:

$\mathcal{S}\left(p_{1}, p_{2}, \ldots\right)=1+2^{0} s_{1}\left(p_{1}\right)+2^{1} s_{2}\left(p_{1}, p_{2}\right)+\cdots+2^{n(n-1) / 2} s_{n}\left(p_{1}, p_{2}, \ldots, p_{n}\right)+\ldots$ 
For the discussion of Schur polynomials, see Sec. 4.2 below.

\section{The Hopf algebra of graphs and umbral graph invariants}

In this section we describe the Hopf algebra structure on the space spanned by graphs. The study of Hopf algebras in combinatorics was initiated in [JR79]. It was then developed in [Sch94, Sch95]. Later it led to the concept of combinatorial Hopf algebra (see [ABS06] and [BM16]) suitable for quasisymmetric functions. Independently the Hopf algebra of graphs was introduced in [CDL94].

Let $\mathcal{G}$ denote the vector space over $\mathbb{C}$ spanned by simple graphs. This vector space is graded,

$$
\mathcal{G}=\mathcal{G}_{0} \oplus \mathcal{G}_{1} \oplus \mathcal{G}_{2} \oplus \ldots
$$

where $\mathcal{G}_{n}$ denotes the finite dimensional vector space spanned by all graphs with $n$ vertices. The product of two graphs is just their disjoint union, while the value of the coproduct $\Delta: \mathcal{G} \rightarrow \mathcal{G} \otimes \mathcal{G}$ on a graph $G$ is obtained as the sum of all terms $G\left(V_{1}\right) \otimes G\left(V_{2}\right)$ where $G\left(V_{1}\right)$ and $G\left(V_{2}\right)$ are two induced subgraphs of $G$ whose vertex sets $V_{1}$ and $V_{2}$ constitute an ordered partition of the vertex set of $G, V(G)=V_{1} \sqcup V_{2}$. Clearly, both the product and the coproduct respect the grading. These operations make the vector space spanned by graphs into a graded bialgebra. This bialgebra is commutative, cocommutative, with a unit given by the empty graph. According to the classical Milnor-Moore theorem it is isomorphic to the algebra of polynomials over its primitive vector space, which is the space of elements $x$ on which the comultiplication $\Delta$ acts as $\Delta(x)=x \otimes 1+1 \otimes x$. Any such bialgebra can be thought of as a Hopf algebra with the antipode acting by multiplication by -1 on the primitive space and counit taking value 1 on the empty graph and 0 on all other graphs.

In the case of $\mathcal{G}$, the reference to the Milnor-Moore theorem can be eliminated by the following observation. Each homogeneous subspace $\mathcal{G}_{n} \subset \mathcal{G}$ admits a representation as a direct sum $\mathcal{G}_{n}=P\left(\mathcal{G}_{n}\right) \oplus D\left(\mathcal{G}_{n}\right)$ of the subspace $P\left(\mathcal{G}_{n}\right)$ of primitive elements and the subspace $D\left(\mathcal{G}_{n}\right)$ of decomposable elements, which is spanned by products of elements having grading smaller than $n$.

Now, the projection $\pi: \mathcal{G}_{n} \rightarrow P\left(\mathcal{G}_{n}\right)$ to the primitive space along the 
space of decomposable elements is given by

$$
\pi(G)=\sum_{V(G)=\bigcup_{\beta \in B} V_{\beta}}(-1)^{|B|-1}(|B|-1) ! \prod_{\beta \in B} G\left(V_{\beta}\right),
$$

where the summation is carried over all unordered partitions $B$ of the set $V(G)$ of vertices of $G$ into disjoint unions of nonempty subsets, $|B|$ is the number of parts, and $G\left(V_{\beta}\right)$ denotes the subgraph of $G$ induced by the subset $V_{\beta} \subset V(G)$ (see [L97], [L00]). Note that the product $\prod_{\beta \in B} G\left(V_{\beta}\right)$ is represented by the graph obtained from $G$ by removing all edges connecting vertices belonging to different parts of the partition.

W. Schmitt's interpretation [Sch94] of the projection to the subspace of primitive elements as the logarithm of the identity mapping allows one to invert Eq. (1) and express any graph as a polynomial in the primitive elements of the form $\pi(G)$ :

$$
G=\sum_{V(G)=\bigcup_{\beta \in B}} \prod_{\beta \in B} \pi\left(G\left(V_{\beta}\right)\right) .
$$

The algebra of polynomials $\mathbb{C}\left[q_{1}, q_{2}, \ldots\right]$ is another commutative and cocommutative Hopf algebra. The multiplicaton acts as the usual multiplication of polynomials and the comultiplication $\Delta$ acts on variables $q_{n}$ as on primitive elements, $\Delta\left(q_{n}\right)=q_{n} \otimes 1+1 \otimes q_{n}$. It becomes graded if we set the degree of $q_{n}$ to be $n$. Thus it has precisely one-dimensional primitive space spanned by $q_{n}$ in each degree $n$.

Definition 3.1 An umbral graph polynomial invariant is an arbitrary homomorphism $F: \mathcal{G} \rightarrow \mathbb{C}\left[q_{1}, q_{2}, \ldots\right]$ of graded Hopf algebras.

The term 'umbral' is justified by the interpretation of the classical umbral calculus as the study of the Hopf algebra of polynomials given in [RR78]. Below, the adjective 'polynomial' will be often omitted since we consider no other kinds of graph invariants. Umbral invariants participate in Theorem 2.3.

Equivalently, umbral invariants can be defined as multiplicative invariants taking primitive elements of $\mathcal{G}$ to linear polynomials (the latter are the primitive elements in the Hopf algebra of polynomials). The condition of respecting comultiplication by $F$ can be reformulated in terms of the binomial property, saying that for any graph $G$,

$F_{G}\left(x_{1}+y_{1}, x_{2}+y_{2}, x_{3}+y_{3}, \ldots\right)=\sum_{V(G)=V_{1} \sqcup V_{2}} F_{G\left(V_{1}\right)}\left(x_{1}, x_{2}, \ldots\right) F_{G\left(V_{2}\right)}\left(y_{1}, y_{2}, \ldots\right)$, 
where the summation on the right runs over all partitions of the set of vertices $V(G)$ into an ordered disjoint union of two subsets. So any umbral graph invariant is uniquely determined by a set of constants $b_{G}$, one for each graph $G$, such that $F_{\pi(G)}=b_{G} q_{|V(G)|}$.

\section{The weighted chromatic polynomial.}

The weighted chromatic polynomial is defined in terms of one more graded Hopf algebra, introduced in [CDL94], namely, the Hopf algebra of weighted graphs $\mathcal{H}$ (it was denoted $\mathcal{W}$ in [CDL94], but in the present paper we reserve the notation $\mathcal{W}$ for the generating function) together with a graded homomorphism $\mathcal{G} \rightarrow \mathcal{H}$. Also it was proved there that $\mathcal{H}$ is isomorphic to $\mathbb{C}\left[q_{1}, q_{2}, \ldots\right]$. Hence we get an umbral graph invariant which makes Theorem 2.1 about the generating function for weighted chromatic polynomials a corollary of the general Theorem 2.3.

The algebra $\mathcal{H}$ is the quotient of the space spanned by weighted graphs modulo the weighted chromatic relation.

Definition 3.2 A weighted graph is a graph $G$ without loops and multiple edges given together with a weight $w: V(G) \rightarrow \mathbb{N}$ that assigns a positive integer to each vertex of the graph.

Ordinary simple graphs can be treated as weighted graphs with the weights of all vertices equal to 1 .

Definition 3.3 The weighted chromatic relation is the set of relations $G=$ $G_{e}^{\prime}+G_{e}^{\prime \prime}$, one for each graph $G$ and an edge $e$ in it, where the graph $G_{e}^{\prime}$ is obtained from $G$ by removing the edge $e$; and $G_{e}^{\prime \prime}$ is obtained from $G$ by a contraction of $e$ such that if a multiple edge arises, it is reduced to a single edge and the weight $w(v)$ of the new vertex $v$ is set up to be equal to the sum of the weights of the two ends of the edge $e$; weights of the other vertices remaining unchanged.

The quotient space of the space spanned by all weighted graphs modulo these relations inherits the multiplication (disjoint union) and the comultiplication (splitting the vertex set into two subsets) and therefore becomes a Hopf algebra. This is $\mathcal{H}$. It is graded by the total weight of a graph, $w(G)=\sum_{v \in V(G)} w(v)$, so that

$$
\mathcal{H}=\mathcal{H}_{0} \oplus \mathcal{H}_{1} \oplus \mathcal{H}_{2} \oplus \ldots,
$$

where the vector space $\mathcal{H}_{n}$ is spanned by all weighted graphs of total weight $n$ modulo weighted chromatic relations. 
It was proved in [CDL94] that the Hopf algebra $\mathcal{H}$ has a one-dimensional primitive space in each grading and thus is isomorphic to $\mathbb{C}\left[q_{1}, q_{2}, \ldots\right]$. Iterating the weighted chromatic relation we can reduce any graph to a linear combination of (disconnected) graphs without edges. In degree $n$, the primitive space $P\left(\mathcal{H}_{n}\right)$ is spanned by the graph which is a single vertex of weight $n$. If we send it to $q_{n}$, then the image of an ordinary graph $G$ (considered as a weighted graph with weights of all vertices equal to 1 ) in $\mathcal{H}$ can be represented by a polynomial in the variables $q_{n}$. This is exactly the weighted chromatic polynomial $W_{G}\left(q_{1}, q_{2}, \ldots\right)$ from the Introduction (p. 2).

A closed formula for $W_{G}\left(q_{1}, q_{2}, \ldots\right)$ is found in [NW99, Corollary 4.4],

$$
W_{G}\left(q_{1}, q_{2}, \ldots\right)=\sum_{E^{\prime} \subseteq E(G)} q_{v_{1}} \ldots q_{v_{k}}(-1)^{\left|E^{\prime}\right|-|V(G)|+k\left(E^{\prime}\right)},
$$

where the sum runs over all subsets $E^{\prime}$ of the edge set $E(G)$, which we may consider as spanning subgraphs of $G, k\left(E^{\prime}\right)$ is the number of connected components of this spanning subgraph, $v_{1}, \ldots, v_{k}$ denoting the numbers of vertices in the connected components.

\section{Example 3.4}

$\underline{\mathbf{n}=\mathbf{1}}$. There is only one graph with one vertex, $G=\bullet$. It is primitive of degree 1 as a weighted graph, whence $W_{\bullet}\left(q_{1}, q_{2}, \ldots\right)=q_{1}$.

$\underline{\mathbf{n}=\mathbf{2}}$. There is only one connected (simple) graph with two vertices, $G=$ $\bullet$ The order of its automorphism group is 2. According to the weighted chromatic relation, it can be represented (as a weighted graph) as $G=\bullet \bullet=$ $(\bullet \bullet)+\underset{2}{\bullet}$, where the weight of a vertex is written underneath, weights 1 being omitted. The first graph is disconnected, whence taken to $q_{1}^{2}$ in $\mathbb{C}\left[q_{1}, q_{2}, \ldots\right]$. The second graph is sent to $q_{2}$. Therefore, $W_{\mathfrak{C}}\left(q_{1}, q_{2}, \ldots\right)=q_{1}^{2}+q_{2}$.

$\underline{\mathbf{n}=3}$. There are two connected simple graphs with three vertices, namely, $\bullet$ and $\triangle$. They have automorphism groups of orders 2 and 6 , respectively. As weighted graphs, modulo the weighted chromatic relation, they can be reduced as follows:

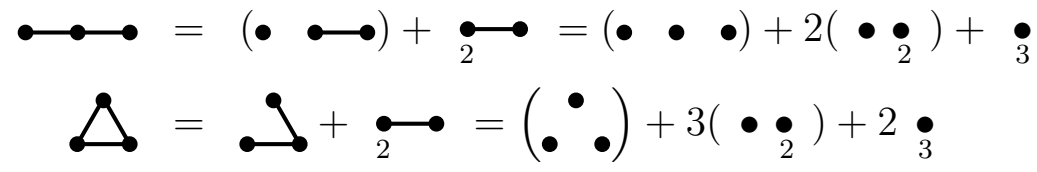

The corresponding weighted chromatic polynomials are therefore

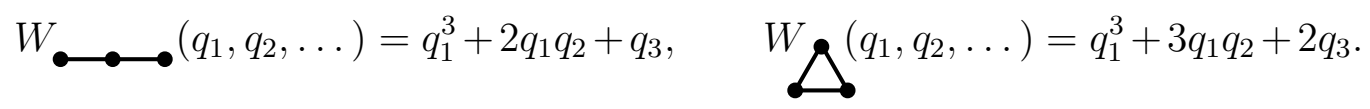


$\underline{\mathbf{n}=4}$. There are six connected simple graphs with four vertices. We arrange the results in the following table.

\begin{tabular}{|c|c|c|}
\hline$G$ & $W_{G}\left(q_{1}, q_{2}, \ldots\right)$ & $\mid$ Aut $(G) \mid$ \\
\hline \hline$\longrightarrow$ & $q_{1}^{4}+3 q_{1}^{2} q_{2}+q_{2}^{2}+2 q_{1} q_{3}+q_{4}$ & 2 \\
\hline & $q_{1}^{4}+3 q_{1}^{2} q_{2}+3 q_{1} q_{3}+q_{4}$ & 6 \\
\hline \\
\hline
\end{tabular}

Consequently, the generating function

$$
\mathcal{W}\left(q_{1}, q_{2}, \ldots\right)=\sum_{\substack{G \text { connected } \\ \text { non-empty }}} \frac{W_{G}\left(q_{1}, q_{2}, \ldots\right)}{|\operatorname{Aut}(G)|}
$$

starts with

$$
\begin{aligned}
\mathcal{W}\left(q_{1}, q_{2}, \ldots\right)= & \frac{1}{1 !} q_{1}+\frac{1}{2 !}\left(q_{1}^{2}+q_{2}\right)+\frac{1}{3 !}\left(4 q_{1}^{3}+9 q_{1} q_{2}+5 q_{3}\right) \\
& +\frac{1}{4 !}\left(38 q_{1}^{4}+144 q_{1}^{2} q_{2}+45 q_{2}^{2}+140 q_{1} q_{3}+79 q_{4}\right)+\ldots,
\end{aligned}
$$

The beginning of its exponent looks like

$$
\begin{aligned}
\mathcal{W}^{\circ}\left(q_{1}, q_{2}, \ldots\right)= & \exp (\mathcal{W})=\sum_{G} \frac{W_{G}\left(q_{1}, q_{2}, \ldots\right)}{|\operatorname{Aut}(G)|} \\
= & 1+\frac{1}{1 !} q_{1}+\frac{1}{2 !}\left(2 q_{1}^{2}+q_{2}\right)+\frac{1}{3 !}\left(8 q_{1}^{3}+12 q_{1} q_{2}+5 q_{3}\right) \\
& +\frac{1}{4 !}\left(64 q_{1}^{4}+192 q_{1}^{2} q_{2}+48 q_{2}^{2}+160 q_{1} q_{3}+79 q_{4}\right)+\ldots
\end{aligned}
$$

The rescaling of variables in Theorem 2.1 gives

$$
q_{1}=p_{1}, \quad q_{2}=2 p_{2}, \quad q_{3}=\frac{2^{3} \cdot 2 !}{5} p_{3}, \quad q_{4}=\frac{2^{6} \cdot 3 !}{79} p_{4}, \quad \ldots
$$


After this substitution we get the function

$$
\begin{aligned}
F\left(p_{1}, p_{2}, \ldots\right)= & \mathcal{W}\left(p_{1}, 2 p_{2}, \frac{16}{5} p_{3}, \frac{384}{79} p_{4}, \ldots\right) \\
= & p_{1}+\frac{1}{2}\left(p_{1}^{2}+2 p_{2}\right)+\frac{1}{6}\left(4 p_{1}^{3}+18 p_{1} p_{2}+16 p_{3}\right) \\
& +\frac{1}{24}\left(38 p_{1}^{4}+288 p_{1}^{2} p_{2}+180 p_{2}^{2}+448 p_{1} p_{3}+384 p_{4}\right)+\ldots,
\end{aligned}
$$

which coincides with the logarithm of the function $\mathcal{S}$ from Theorem 2.4.

One may easily check that $\frac{\partial^{2} F}{\partial p_{2}^{2}}=15+\ldots, \quad$ while

$$
\frac{\partial^{2} F}{\partial p_{1} \partial p_{3}}-\frac{1}{2}\left(\frac{\partial^{2} F}{\partial p_{1}^{2}}\right)^{2}-\frac{1}{12} \frac{\partial^{4} F}{\partial p_{1}^{4}}=\frac{56}{3}-\frac{1}{2}-\frac{19}{6}+\cdots=15+\ldots
$$

giving thus an evidence that we get a solution of the first KP equation.

\section{The Abel polynomial.}

Example 3.5 This example is similar to 3.4. We will follow it case by case.

$\underline{\mathbf{n}=\mathbf{1}}$. For $G=\bullet$, we have $A_{\bullet}\left(q_{1}, q_{2}, \ldots\right)=q_{1}$.

$\underline{\mathbf{n}=\mathbf{2}}$. For $G=\bullet$, there are two spanning forests, $(\bullet \bullet)$ and the graph itself $\bullet$. Therefore, $A \bullet\left(q_{1}, q_{2}, \ldots\right)=q_{1}^{2}+2 q_{2}$.

$\underline{\mathbf{n}=3}$. There are two connected simple graphs with three vertices,

and $\triangle$, with automorphism groups of order 2 and 6, respectively. For the first one, $\bullet \bullet$, there are four spanning forests

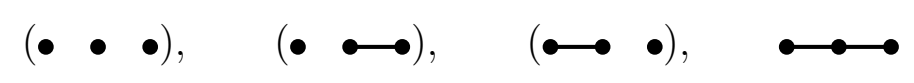

which contribute the following monomials to the Abel polynomial:

$$
q_{1}^{3}, \quad 2 q_{1} q_{2}, \quad 2 q_{1} q_{2}, \quad 3 q_{3} .
$$

Thus $A \longrightarrow\left(q_{1}, q_{2}, \ldots\right)=q_{1}^{3}+4 q_{1} q_{2}+3 q_{3}$.

For the triangle, $\triangle$, there are seven spanning forests

$$
(\therefore)(\therefore),(\therefore),(\therefore) \sim \wedge
$$


Each of the last three spanning trees contributes $3 q_{3}$. The previous three spanning forests contribute $2 q_{1} q_{2}$ each. Hence the Abel polynomial of the triangle is $A\left(q_{1}, q_{2}, \ldots\right)=q_{1}^{3}+6 q_{1} q_{2}+9 q_{3}$.

$\underline{\mathbf{n}=4}$. For the six connected simple graphs with four vertices, we collect the results in the following table.

\begin{tabular}{|c|c|c|}
\hline$G$ & $A_{G}\left(q_{1}, q_{2}, \ldots\right)$ & $\mid$ Aut $(G) \mid$ \\
\hline \hline \\
\hline
\end{tabular}

Thus the generating function

$$
\mathcal{A}\left(q_{1}, q_{2}, \ldots\right)=\sum_{\substack{G \text { connected } \\ \text { non-empty }}} \frac{A_{G}\left(q_{1}, q_{2}, \ldots\right)}{|\operatorname{Aut}(G)|}
$$

starts with

$$
\begin{aligned}
\mathcal{A}\left(q_{1}, q_{2}, \ldots\right)= & \frac{1}{1 !} q_{1}+\frac{1}{2 !}\left(q_{1}^{2}+2 q_{2}\right)+\frac{1}{3 !}\left(4 q_{1}^{3}+18 q_{1} q_{2}+18 q_{3}\right) \\
& +\frac{1}{4 !}\left(38 q_{1}^{4}+288 q_{1}^{2} q_{2}+180 q_{2}^{2}+504 q_{1} q_{3}+512 q_{4}\right)+\ldots .
\end{aligned}
$$

The rescaling of variables in Theorem 2.2 has the form

$$
q_{1}=p_{1}, \quad q_{2}=p_{2}, \quad q_{3}=\frac{8}{9} p_{3}, \quad q_{4}=\frac{3}{4} p_{4}, \quad \ldots
$$


After the substitution we come to the same series

$$
\begin{aligned}
F\left(p_{1}, p_{2}, \ldots\right)= & \mathcal{A}\left(p_{1}, p_{2}, \frac{8}{9} p_{3}, \frac{3}{4} p_{4}, \ldots\right) \\
= & p_{1}+\frac{1}{2}\left(p_{1}^{2}+2 p_{2}\right)+\frac{1}{6}\left(4 p_{1}^{3}+18 p_{1} p_{2}+16 p_{3}\right) \\
& +\frac{1}{24}\left(38 p_{1}^{4}+288 p_{1}^{2} p_{2}+180 p_{2}^{2}+448 p_{1} p_{3}+384 p_{4}\right)+\ldots,
\end{aligned}
$$

as before. It is interesting to note that for an individual graph $G$, the rescalings of the variables under consideration give different polynomials $W_{G}\left(p_{1}, 2 p_{2}, \frac{16}{5} p_{3}, \frac{384}{79} p_{4}, \ldots\right)$ and $A_{G}\left(p_{1}, p_{2}, \frac{8}{9} p_{3}, \frac{3}{4} p_{4}, \ldots\right)$. For example, for the six graphs with four vertices from our tables, the coefficients of $p_{4}$ in $W_{G}$ will be $\frac{384}{79}, \frac{384}{79}, \frac{768}{79}, \frac{1152}{79}, \frac{1536}{79}, \frac{2304}{79}$, while in $A_{G}$ they will be $3,3,9,12,24,48$. However, after division by $|\operatorname{Aut}(G)|$ and summation to the generating functions they become equal:

$$
\begin{aligned}
\frac{1}{24} \cdot \frac{12 \cdot 384+4 \cdot 384+12 \cdot 768+3 \cdot 1152+6 \cdot 1536+2304}{79}=\frac{384}{24} & =16 \\
\frac{1}{24} \cdot(12 \cdot 3+4 \cdot 3+12 \cdot 9+3 \cdot 12+6 \cdot 24+48) & =16 .
\end{aligned}
$$

Theorem 3.1 The Abel polynomial $A: \mathcal{G} \rightarrow \mathbb{C}\left[q_{1}, q_{2}, \ldots\right]$ is an umbral graph invariant.

It is clear that the Abel polynomial is homogeneous and multiplicative. In order to prove that the mapping $A: \mathcal{G} \rightarrow \mathbb{C}\left[q_{1}, q_{2}, \ldots\right]$ respects comultiplication, it suffices to show that the Abel polynomial possesses the binomial property, that is, that it satisfies the relation

$$
A_{G}\left(x_{1}+y_{1}, x_{2}+y_{2}, \ldots\right)=\sum_{V(G)=V_{1} \sqcup V_{2}} A_{G\left(V_{1}\right)}\left(x_{1}, x_{2}, \ldots\right) A_{G\left(V_{2}\right)}\left(y_{1}, y_{2}, \ldots\right),
$$

where the summation on the right runs over all partitions of the set of vertices $V(G)$ into an ordered disjoint union of two subsets.

The binomial property is satisfied because of the following argument. Consider a spanning forest in the graph $G$. The contribution of this spanning forest to the expression on the left is

$$
\prod_{i=1}^{\infty}\left(i\left(x_{i}+y_{i}\right)\right)^{k_{i}},
$$

where $k_{i}$ is the number of trees with $i$ vertices in the forest, $k_{i}=0$ for $i$ large enough. These $k_{i}$ trees can be distributed between the two subsets $V^{\prime}, V^{\prime \prime}$ 
of the partition of the set of vertices $V(G)$. There are $\left(\begin{array}{c}k_{i} \\ \ell_{i}\end{array}\right)$ ways to choose $\ell_{i}$ trees for the first part thus leaving $k_{i}-\ell_{i}$ trees for the second part, for $\ell_{i}=0,1,2, \ldots, k_{i}$. Hence the binomial property for the Abel polynomial follows from the usual binomial identity

$$
\left(x_{i}+y_{i}\right)^{k_{i}}=\sum\left(\begin{array}{c}
k_{i} \\
\ell_{i}
\end{array}\right) x_{i}^{\ell_{i}} y_{i}^{k_{i}-\ell_{i}} .
$$

Remark 3.6 Suppose we have a multiplicative polynomial graph invariant taking values in the ring $\mathbb{C}[x]$ of polynomials in a single variable $x$. Suppose also that the value of this invariant on any primitive element in the Hopf algebra of graphs is a linear polynomial, that is, $x$ with some coefficient. Then such a graph invariant admits a canonical umbralization. Namely, we can associate to this graph invariant a homomorphism from the Hopf algebra of graphs to the Hopf algebra $\mathbb{C}\left[q_{1}, q_{2}, \ldots\right]$ of polynomials in infinitely many variables, which is determined uniquely by the requirement that its value on the projection $\pi(G)$ of an arbitrary graph $G$ to the subspace of primitive elements is $b_{G} q_{n}$, where $n=|V(G)|$ is the number of vertices of $G$, and $b_{G}$ is the coefficient of $x$ in the value of the given invariant on $G$.

For example, Stanley's symmetrized chromatic polynomial is the umbralization of the ordinary chromatic polynomial. Similarly, the Abel polynomial we introduce is the umbralization of the one-variable polynomial graph invariant whose coefficient of $x^{k}$, for a given graph $G$, is the number of rooted forests in $G$ consistsing of $k$ trees.

\section{General information on the Kadomtsev- Petviashvili integrable hierarchy}

In this section, we reproduce necessary information about the KadomtsevPetviashvili (KP) integrable hierarchy of partial differential equations. A more detailed explanation, suitable for the first acquaintance, can be found, for example, in [KL15].

\section{$4.1 \tau$-functions and solutions to the KP hierarchy}

The KP integrable hierarchy of partial differential equations is best described in terms of its solutions and $\tau$-functions. The latter are the exponents of the former. In 1983, Sato [S83] shown that the $\tau$-functions of the KP-hierarchy are in a natural one-to-one correspondence with semi-infinite planes in an infinite dimensional vector space of Laurent polynomials. 
Let $V$ be the vector space of Laurent polynomials in the variable $z$. The semi-infinite Grassmannian $G\left(\frac{\infty}{2}, V\right)$ (to be more precise, an open dense cell in this Grassmannian) consists of decomposable vectors in $P \Lambda^{\frac{\infty}{2}} V$, i.e., of vectors of the form

$$
\beta_{1}(z) \wedge \beta_{2}(z) \wedge \beta_{3}(z) \wedge \ldots,
$$

where $\beta_{i}$ are Laurent series in $z$, and we have

$$
\beta_{i}(z)=z^{-i}+c_{i 1} z^{-i+1}+c_{i 2} z^{-i+2}+\ldots
$$

The semi-infinite wedge product $\Lambda^{\frac{\infty}{2}} V$ of the vector space $V$ is, by definition, the vector space spanned by the vectors

$$
v_{\mu}=z^{m_{1}} \wedge z^{m_{2}} \wedge z^{m_{3}} \wedge \ldots, \quad m_{1}>m_{2}>m_{3}>\ldots, \quad m_{i}=\mu_{i}-i,
$$

where $\mu$ is a partition, $\mu=\left(\mu_{1}, \mu_{2}, \mu_{3}, \ldots\right), \mu_{1} \geq \mu_{2} \geq \mu_{3} \geq \ldots$, having all but finitely many parts equal to 0 . In particular, $m_{i}=-i$ for all $i$ large enough.

The empty partition corresponds to the vacuum vector

$$
v_{\emptyset}=z^{-1} \wedge z^{-2} \wedge z^{-3} \wedge \ldots
$$

Similarly,

$v_{1^{1}}=z^{0} \wedge z^{-2} \wedge z^{-3} \wedge \ldots, \quad v_{2^{1}}=z^{1} \wedge z^{-2} \wedge z^{-3} \wedge \ldots, \quad v_{1^{2}}=z^{0} \wedge z^{-1} \wedge z^{-3} \wedge \ldots$, and so on.

Numbering of the basic vectors in the semi-infinite wedge product $\Lambda^{\frac{\infty}{2}} V$ (the space of fermions) by partitions of nonnegative integers establishes its natural vector space isomorphism with the space of bosons, that is, power series in infinitely many variables $p_{1}, p_{2}, \ldots$ It is called the boson-fermion isomorphism. This isomorphism takes a basic vector $v_{\mu}$ to the Schur polynomial $s_{\mu}=s_{\mu}\left(p_{1}, p_{2}, \ldots\right)$. The latter is a quaihomogeneous polynomial of degree $|\mu|$ in the variables $p_{i}$ (by definition, the degree of $p_{i}$ is $i$ ). Schur polynomials form an additive basis of the vector space of power series in the variables $p_{i}$.

Definition 4.1 The Hirota equations are the Plücker equations of the embedding of the semi-infinite Grassmannian into the projectivized semi-infinite wedge product $P \Lambda^{\frac{\infty}{2}} V$. Solutions of the Hirota equations (that is, semiinfinite planes) are called $\tau$-functions of the KP hierarchy. 
Let us represent a power series in $p_{1}, p_{2}, \ldots$ as a linear combination

$$
\sum_{\nu} c_{\nu} s_{\nu}\left(p_{1}, p_{2}, \ldots\right)
$$

of Schur polynomials. The Hirota equations are quadratic in the coefficients $c_{\nu}$ of these linear combinations.

Definition 4.2 The form the Hirota equations take for the logarithm of a $\tau$-function are called the Kadomtsev-Petviashvili hierarchy equations.

In other words, each $\tau$-function of the KP hierarchy can be obtained as the result of the following procedure:

- take a semi-infinite plane $\beta_{1}(z) \wedge \beta_{2}(z) \wedge \ldots$ in $V$;

- using the Laurent expansion of $\beta_{i}$ represent the corresponding point in the semi-infinite Grassmannian as a linear combination of the basic vectors $v_{\kappa}$ and multiply by a constant in order to make 1 the coefficient of the vacuum-vector $v_{\emptyset}$;

- replace each vector $v_{\kappa}$ in the resulting linear combination with the corresponding Schur polynomial $s_{\kappa}\left(p_{1}, p_{2}, \ldots\right)$; this series in $p_{1}, p_{2}, \ldots$ is the desired $\tau$-function.

Logarithms of $\tau$-functions are solutions to the KP hierarchy.

\subsection{Schur polynomials and constructions of combinato- rial $\tau$-functions}

For $\lambda$ being partitions of a given non negative integer $n$, the Schur polynomials $s_{\lambda}$ form an additive basis in the vector space of symmetric polynomials in infinitely many variables $x_{1}, x_{2}, \ldots$ In particular, the one-part Schur polynomial $s_{n^{1}}=s_{n}$ is defined as the complete homogeneous symmetric polynomial, which is the sum of all monomials of homogeneous degree $n$ in variables $x_{1}, x_{2}, \ldots$.

After substituting for $p_{i}$ the power sum functions

$$
p_{i}=x_{1}^{i}+x_{2}^{i}+\ldots, \quad i=1,2, \ldots,
$$

the Schur polynomials turn into an additive basis in the vector space of quasihomogeneous polynomials in $p_{1}, \ldots, p_{n}$, with the weight of $p_{i}$ taken to be $i$, for $i=1,2, \ldots$. 
The expression of the one-part Schur polynomial $s_{n}$ in variables $p_{1}, p_{2}, \ldots$ can be found from the generating function

$$
\begin{aligned}
\sum_{n=0}^{\infty} s_{n} & =e^{\sum_{k=1}^{\infty} \frac{p_{k}}{k}} \\
& =1+p_{1}+\frac{1}{2 !}\left(p_{1}^{2}+p_{2}\right)+\frac{1}{3 !}\left(p_{1}^{3}+3 p_{1} p_{2}+2 p_{3}\right)+\ldots
\end{aligned}
$$

For arbitrary partition $\lambda=\left(\lambda_{1}, \lambda_{2}, \ldots, \lambda_{l}\right), \lambda_{1} \geq \lambda_{2} \geq \cdots \geq \lambda_{l}$, the Schur polynomial $s_{\lambda}$ can be given by the Jacobi-Trudi formula

$$
s_{\lambda}=\operatorname{det}\left(\begin{array}{ccccc}
s_{\lambda_{1}} & s_{\lambda_{1}+1} & s_{\lambda_{1}+2} & \ldots & s_{\lambda_{1}+l-1} \\
s_{\lambda_{2}-1} & s_{\lambda_{2}} & s_{\lambda_{1}+1} & \ldots & s_{\lambda_{2}+l-2} \\
\ldots & \ldots & \ldots & \ldots & \ldots \\
s_{\lambda_{l}-l+1} & s_{\lambda_{l}-l+2} & s_{\lambda_{l}-l+3} & \ldots & s_{\lambda_{l}}
\end{array}\right) \text {. }
$$

The Schur polynomials constitute a convenient language for describing formal $\tau$-functions as well as solutions to the KP hierarchy. In particular, the following statement is true.

Proposition 4.3 Any linear combination

$$
1+c_{1} s_{1}+c_{2} s_{2}+\ldots
$$

of one-part Schur polynomials with constant coefficients $c_{n}$ is a $\tau$-function for the KP hierarchy.

Indeed, consider the semi-infinite plane $\beta_{1}(z) \wedge z^{-2} \wedge z^{-3} \wedge \ldots$, where $\beta_{1}(z)=z^{-1}+c_{1} z^{0}+c_{2} z^{1}+c_{3} z^{2}+\ldots$ Since

$$
\beta_{1}(z) \wedge z^{-2} \wedge z^{-3} \wedge \cdots=v_{\emptyset}+c_{1} v_{1^{1}}+c_{2} v_{2^{1}}+\ldots,
$$

this plane is taken, under the boson-fermion correspondence, to the power series in the Proposition.

\section{Proof of the main theorems}

In this section, we give the proofs of main theorems stated in Sec. 2 . 


\subsection{Proof of Theorem 2.3}

Define the universal umbral graph invariant $G \mapsto U_{G}\left(q_{1}, q_{2}, \ldots\right)$ as the multiplicative graph invariant taking value $b_{G} q_{|V(G)|}$ on the primitive element $\pi(G)$, which is the projection of a graph $G$ to the subspace of primitive elements along the subspace of decomposable elements in $\mathcal{G}$, for all connected graphs $G$. The invariant $U_{G}$ takes values in the ring of polynomials in the variables $q_{1}, q_{2}, \ldots$ whose coefficients are polynomials in the variables $b_{H}$, for all connected graphs $H$. (Of course, for a given graph $G$, the coefficients of the polynomial $U_{G}$ depend only on those indeterminates $b_{H}$ such that $H$ is an induced subgraph of $G$ ). We call the invariant $U$ universal because the projections $\pi(G)$ of connected graphs to the subspace of primitive elements form a basis in the space of primitive elements of $\mathcal{G}$, whence any umbral graph invariant with values in the ring $\mathbb{C}\left[q_{1}, q_{2}, \ldots\right]$ can be induced from $U$ by choosing appropriate values of $b_{G}$, for all connected graphs $G$.

Introduce two generating functions

$$
\begin{aligned}
\mathcal{U}^{\circ}\left(q_{1}, q_{2}, \ldots\right) & =\sum_{G} \frac{U_{G}\left(q_{1}, q_{2}, \ldots\right)}{|\operatorname{Aut}(G)|} \\
\mathcal{U}\left(q_{1}, q_{2}, \ldots\right) & =\sum_{G \text { connected }} \frac{U_{G}\left(q_{1}, q_{2}, \ldots\right)}{|\operatorname{Aut}(G)|} .
\end{aligned}
$$

As above, we have

$$
\mathcal{U}=\exp \left(\mathcal{U}^{\circ}\right)
$$

For $k=0,1,2, \ldots$, denote by

$$
u_{k}=\sum_{\substack{G \text { connected } \\|V(G)|=k}} \frac{b_{G}}{|\operatorname{Aut}(G)|}
$$

the coefficient of the monomial $q_{k}$ in either $\mathcal{U}$ or $\mathcal{U}^{\circ}$. As well, denote by

$$
\begin{aligned}
& \mathcal{U}_{k}^{\circ}\left(q_{1}, q_{2}, \ldots\right)=\sum_{G,|V(G)|=k} \frac{U_{G}\left(q_{1}, q_{2}, \ldots\right)}{|\operatorname{Aut}(G)|} \\
& \mathcal{U}_{k}\left(q_{1}, q_{2}, \ldots\right)=\sum_{\substack{G \text { connected } \\
|V(G)|=k}} \frac{U_{G}\left(q_{1}, q_{2}, \ldots\right)}{|\operatorname{Aut}(G)|}
\end{aligned}
$$

the $k$ th homogeneous parts of the generating functions $\mathcal{U}^{\circ}$ for all graphs and $\mathcal{U}$ for connected graphs, respectively, so that

$$
\begin{aligned}
\mathcal{U}^{\circ}\left(q_{1}, q_{2}, \ldots\right) & =1+\mathcal{U}_{1}^{\circ}\left(q_{1}, q_{2}, \ldots\right)+\mathcal{U}_{2}^{\circ}\left(q_{1}, q_{2}, \ldots\right)+\ldots \\
\mathcal{U}\left(q_{1}, q_{2}, \ldots\right) & =\mathcal{U}_{1}\left(q_{1}, q_{2}, \ldots\right)+\mathcal{U}_{2}\left(q_{1}, q_{2}, \ldots\right)+\ldots
\end{aligned}
$$


Introduce notation

$$
Q_{k}\left(q_{1}, q_{2}, \ldots\right)=s_{k}\left(\frac{u_{1}}{0 ! 2^{0}} q_{1}, \frac{u_{2}}{1 ! 2^{1}} q_{2}, \frac{u_{3}}{2 ! 2^{3}} q_{3}, \ldots\right),
$$

that is, $Q_{k}\left(q_{1}, q_{2}, \ldots\right)$ is the result of the substitution $p_{i}=\frac{u_{i}}{(i-1) ! 2} q_{2}^{\left(\begin{array}{c}i \\ 2\end{array}\right)} q_{i}$ into the one-part Schur function $s_{k}\left(p_{1}, p_{2}, \ldots, p_{k}\right)$.

Theorem 5.1 For each $k=0,1,2, \ldots$, we have

$$
\mathcal{U}_{k}^{\circ}\left(q_{1}, q_{2}, \ldots\right)=2^{\left(\begin{array}{c}
k \\
2
\end{array}\right)} Q_{k}\left(q_{1}, q_{2}, \ldots, q_{k}\right)
$$

so that

$$
\mathcal{U}^{\circ}\left(q_{1}, q_{2}, \ldots\right)=Q_{0}+2^{0} Q_{1}+2^{1} Q_{2}+2^{3} Q_{3}+2^{6} Q_{4}+\ldots
$$

This means that after the specified rescaling of the variables,

$$
q_{k}=\frac{2^{\left(\begin{array}{c}
k \\
2
\end{array}\right)(k-1) !}}{u_{k}} p_{k}, \quad k=1,2, \ldots
$$

the generating function $\mathcal{U}\left(q_{1}, q_{2}, \ldots\right)$ becomes a linear combination of onepart Schur functions, namely, the function $\mathcal{S}\left(p_{1}, p_{2}, \ldots\right)$ from Theorem 2.4, whence, according to Proposition 4.3, a $\tau$-function for the KP hierarchy.

In order to prove the theorem, we will need the following statement.

Set $b_{G}=0$ for disconnected graphs $G$.

Now, Eq. (2) shows that for any (either connected or disconnected) graph $G$, we have

$$
U_{G}\left(q_{1}, q_{2}, \ldots\right)=\sum_{V(G)=\sqcup_{\alpha} V_{\alpha}} \prod_{\alpha} b_{G\left(V_{\alpha}\right)} q_{\left|V_{\alpha}\right|}
$$

where the summation is carried over all unordered partitions of the set $V(G)$ of vertices of $G$ into disjoint unions of subsets, and $G\left(V_{\alpha}\right)$ denotes the subgraph of $G$ induced by the subset $V_{\alpha} \subset V(G)$.

In order to prove Theorem 5.1, let us replace summation over all graphs with $k$ vertices with summing over all spanning subgraphs of the complete graph $K_{k}$ on $k$ vertices. Obviously, for a graph $G$ with $k$ vertices we have

$$
\frac{U_{G}\left(q_{1}, q_{2}, \ldots\right)}{|\operatorname{Aut}(G)|}=\frac{1}{k !} \sum_{\substack{\left.E \subset E\left(K_{k}\right) \\ K_{k}\right|_{E}=G}} U_{\left.K_{k}\right|_{E}}\left(q_{1}, q_{2}, \ldots\right),
$$


where the summation is carried over all spanning subgraphs $\left.K_{k}\right|_{E}$ of $K_{k}$ that are isomorphic to $G$. Hence,

$$
\mathcal{U}_{k}^{\circ}\left(q_{1}, q_{2}, \ldots\right)=\frac{1}{k !} \sum_{E \subset E\left(K_{k}\right)} U_{\left.K_{k}\right|_{E}}\left(q_{1}, q_{2}, \ldots\right),
$$

and we have replaced summation over isomorphism classes of graphs with $k$ vertices with summation over subsets in the set of edges of the complete graph with $k$ vertices.

Making use of Eq. (4) we obtain

$$
\mathcal{U}_{k}^{\circ}\left(q_{1}, q_{2}, \ldots\right)=\frac{1}{k !} \sum_{V\left(K_{k}\right)=\sqcup_{\alpha} V_{\alpha}} 2^{\left(\begin{array}{c}
k \\
2
\end{array}\right)-\sum_{\alpha}\left(\begin{array}{c}
\left|V_{\alpha}\right| \\
2
\end{array}\right)} \prod_{\alpha} \sum_{E \subset E\left(K_{k}\left(V_{\alpha}\right)\right)} b_{K_{k}\left(V_{\alpha}\right) \mid{ }_{E}} q_{\left|V_{\alpha}\right|} .
$$

Here $\left(\begin{array}{l}k \\ 2\end{array}\right)-\sum_{\alpha}\left(\begin{array}{c}\left|V_{\alpha}\right| \\ 2\end{array}\right)$ is the number of the edges of $K_{k}$ connecting vertices belonging to distinct subsets $V_{\alpha}$. Dividing both sides of the last equation by $2^{\left(\begin{array}{c}k \\ 2\end{array}\right)}$ we obtain

$$
\begin{aligned}
2^{-\left(\begin{array}{c}
k \\
2
\end{array}\right) \mathcal{U}_{k}^{\circ}\left(q_{1}, q_{2}, \ldots\right)} & =\frac{1}{k !} \sum_{V\left(K_{k}\right)=\sqcup_{\alpha} V_{\alpha}} \prod_{\alpha} 2^{-\left(\begin{array}{c}
\left|V_{\alpha}\right| \\
2
\end{array}\right)}\left|V_{\alpha}\right| ! u_{\left|V_{\alpha}\right|} q_{\left|V_{\alpha}\right|} \\
& =\frac{1}{k !} \sum_{V\left(K_{k}\right)=\sqcup_{\alpha} V_{\alpha}} \prod_{\alpha}\left(\left|V_{\alpha}\right|-1\right) ! p_{\left|V_{\alpha}\right|} .
\end{aligned}
$$

The right-hand side of the last equation is independent of the variables $b_{G}$ and coincides with the definition of the one-part Schur function $s_{k}=$ $s_{k}\left(p_{1}, p_{2}, \ldots\right)$, which proves the theorem.

\subsection{Specialization to weighted chromatic polynomial}

Theorem 2.1 for the weighted chromatic polynomial $W_{G}$ follows from the main theorem, since the weighted chromatic polynomial is an example of umbral graph invariant. For this invariant, the sequence

$$
1,1,5,79,3377, \ldots
$$

of the values $c_{1}, c_{2}, c_{3}, \ldots$ can be described as follows. The exponent of the exponential generating function for the alternating sequence

$$
x-\frac{1}{2 ! 2^{1}} x^{2}+\frac{5}{3 ! 2^{3}} x^{3}-\frac{79}{4 ! 2^{6}} x^{4}+\frac{3377}{5 ! 2^{10}} x^{5}-\ldots
$$

is

$$
1+x+\frac{1}{2 ! 2^{1}} x^{2}+\frac{1}{3 ! 2^{3}} x^{3}+\frac{1}{4 ! 2^{6}} x^{4}+\frac{1}{5 ! 2^{10}} x^{5}+\ldots
$$


In other words, we have the recursion

$$
(-1)^{n+1} c_{n}=1-\sum_{k=1}^{n-1}(-1)^{k+1} 2^{k(n-k)}\left(\begin{array}{c}
n-1 \\
k-1
\end{array}\right) c_{k} .
$$

In order to prove this statement, let us compute explicitly the coefficient $C_{k}$ of $x^{k}$ in the exponent

$$
\exp \left(\sum_{i=1}^{\infty} \frac{(-1)^{i-1} c_{i}}{i ! 2^{\left(\begin{array}{c}
i \\
2
\end{array}\right)}} x^{i}\right)
$$

This exponent is the result of substitution $p_{i}=\frac{(-1)^{i-1} c_{i}}{\left.(i-1) ! 2^{(} 2^{2}\right)} x^{i}$ into $\exp \sum_{i=1}^{\infty}\left(p_{i} / i\right)$, therefore, $C_{k} x^{k}$ is the value of the Schur function $s_{k}$ at the same substitution. By Theorem 5.1, we get

$$
C_{k}=\left.2^{-\left(\begin{array}{c}
k \\
2
\end{array}\right)} \mathcal{W}_{k}^{\circ}\left(q_{1}, q_{2}, \ldots\right)\right|_{q_{i}=(-1)^{i-1}},
$$

where $\mathcal{W}_{k}^{\circ}$ is the degree $k$ quasihomogeneous summand of $\mathcal{W}^{\circ}$. Now, following the proof of Theorem 5.1, we get

$$
C_{k}=\left.2^{-\left(\begin{array}{c}
k \\
2
\end{array}\right)} \frac{1}{k !} \sum_{E \subset E\left(K_{k}\right)} W_{\left.K_{k}\right|_{E}}\left(q_{1}, q_{2}, \ldots\right)\right|_{q_{i}=(-1)^{i-1}},
$$

where the summation is carried over all spanning subgraphs $\left.K_{k}\right|_{E}$ of $K_{k}$. Up to this point, similar computations can be applied to any umbral graph invariant. Now, for the particular case of the invariant $W_{G}$, we get by (3),

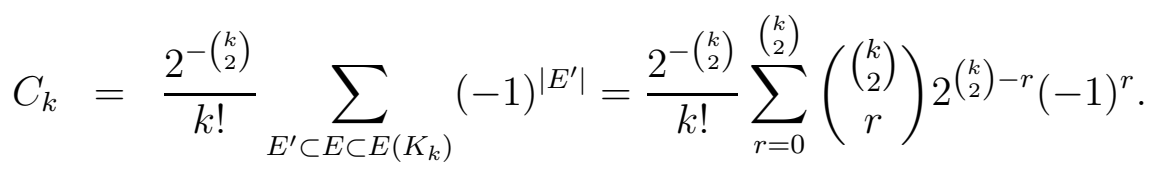

The first factor inside the last summation is the number of ways to choose a subgraph $\left.K_{k}\right|_{E^{\prime}}$ with $r$ edges, and the second factor is the number of ways to choose a subgraph $\left.K_{k}\right|_{E}$ containing $\left.K_{k}\right|_{E^{\prime}}$. Thus, we obtain finally

$$
C_{k}=\frac{1}{k !} \sum_{r=0}^{\left(\begin{array}{c}
k \\
2
\end{array}\right)}\left(\begin{array}{c}
\left(\begin{array}{c}
k \\
2
\end{array}\right) \\
r
\end{array}\right)(-2)^{-r}=\frac{1}{k !}\left(1-\frac{1}{2}\right)^{\left(\begin{array}{c}
k \\
2
\end{array}\right)}=\frac{1}{k ! 2^{\left(\begin{array}{c}
k \\
2
\end{array}\right)}}
$$

This is what we claimed. 


\subsection{Specialization to Abel polynomial}

We have proved already that the Abel polynomial possesses the binomial property and is therefore umbral. Hence it suffices to find the sequence $\left\{a_{n}\right\}$ defined as

$$
a_{n}=\sum_{G} \frac{\left[q_{n}\right] A_{G}}{|\operatorname{Aut} G|}
$$

where the summation is carried over all connected graphs with $n$ vertices. As above, the last summation can be replaced by summation over all spanning subgraphs of the complete graph $K_{n}$. The contribution of any spanning tree into this sum is $2^{(n-1)(n-2) / 2} \cdot n$, since there are $n$ ways to choose a root in the tree, and each tree enters exactly $2^{(n-1)(n-2) / 2}$ spanning (and necessarily connected) subgraphs of $K_{n}$ (this is the number of ways to add other edges to the tree to obtain a spanning subgraph containing this tree). Since, by Caley's theorem, the number of spanning trees in $K_{n}$ is $n^{n-2}$, we obtain the desired equality

$$
a_{n}=2^{\left(\begin{array}{c}
n-1 \\
2
\end{array}\right)} n^{n-1} \text {. }
$$

\section{References}

[A134531] The On-Line Encyclopedia of Integer Sequences, http://oeis.org/A134531.

[Ab826] N. H. Abel, Beweis eines Ausdruckes, von welchem die Binomialformel ein einzelner Fall ist, Journal für die reine und angewandte Mathematik 1 159-160 (1826).

[ABS06] M. Aguiar, N. Bergeron, F. Sottile, Combinatorial Hopf algebras and generalized Dehn-Sommerville relations, Compositio Math. 142 130 (2006).

[BM16] S. Billey, P. McNamara, The contributions of Stanley to the fabric of symmetric and quasisymmetric functions, in The mathematical legacy of Richard P. Stanley, Editors P. Herch, T. Lam, P. Pylyavskyy, V. Reiner 83-104 (2016).

[CS06] P. Chebotarev, E. Shamis, Matrix-Forest Theorems, arXiv:math/0602575 [math.CO].

[CDL94] S. Chmutov, S. Duzhin, S. Lando, Vassiliev knot invariants III. Forest algebra and weighted graphs, Advances in Soviet Mathematics 21 135-145 (1994). 
[JR79] S. Joni, G.-C. Rota, Coalgebras and bialgebras in combinatorics, Stud. Appl. Math. 61(2) 93-139 (1979).

[KL15] M. Kazarian, S. Lando, Combinatorial solutions to integrable hierarchies, Russ. Math. Surveys, 70(3) 453-482 (2015).

[Kn13] O. Knill, Counting rooted forests in a network, arXiv:1307.3810 [math.SP].

[L97] S. K. Lando, On primitive elements in the bialgebra of chord diagrams, in: Topics in singularity theory, 167-174, Amer. Math. Soc. Transl. Ser. 2, 180, Adv. Math. Sci., 34, Amer. Math. Soc., Providence, RI, 1997

[L00] S. K. Lando, On a Hopf algebra in graph theory, J. Comb. Theory, Ser. B, vol. 80 (2000), 104-121.

[NW99] S. Noble, D. Welsh, A weighted graph polynomial from chromatic invariants of knots, Annales de l'institut Fourier 49(3) 1057-1087 (1999).

[O00] A. Okounkov, Toda equations for Hurwitz numbers, Math. Res. Lett. 7(4) 447-453 (2000).

[RR78] S. Roman, G.-C. Rota, The Umbral Calculus, Adv. Math. 27, 95188 (1978)

[RST97] G-C. Rota, J. Shen, B. Taylor, All Polynomials of Binomial Type Are Represented by Abel Polynomials, Annali della Scuola Normale Superiore di Pisa - Classe di Scienze, Série 4 25(3-4): 731-738 (1997).

[S83] M. Sato and Y. Sato, Soliton equations as dynamical systems on infinite dimensional Grassmann manifolds, in: Nonlinear partial differential equations in applied science (Tokyo 1982), North-HollandMath. Stud., vol. 81, North- Holland, Amsterdam 1983, pp. 259-271.

[Sch94] W. R. Schmitt, Incidence Hopf algebras, Journal of Pure and Applied Algebra 96 299-330 (1994).

[Sch95] W. R. Schmitt, Hopf algebra methods in graph theory, Journal of Pure and Applied Algebra 101(1) 77-90 (1995).

[St95] R. Stanley, A symmetric function generalization of the chromatic polynomial of a graph, Advances in Math. 111(1) 166-194 (1995). 